\title{
LA PRECARIZACIÓN DEL TRABAJO DOCENTE EN EL SISTEMA EDUCACIONAL CHILENO
}

\author{
Paulina Sofía Santibáñez Cavieres ${ }^{1}$ \\ Gilda Katherine Muñoz Cifuentes ${ }^{2}$
}

\begin{abstract}
RESUMEN: El actual panorama social de Chile es complejo, caracterizado por la implementación, desde la década de los 80 , de políticas económicas y sociales basadas en la ideología neoliberal. Lo anterior generó que derechos fundamentales - como salud, educación, seguridad social - fueran abandonados por un Estado que se manifiesta como subsidiario y no como garante de éstos, teniendo como objetivo central fortalecer el libre mercado en todas las áreas posibles. Considerando la actual crisis que este proceso de neoliberalización ha producido en la educación chilena y en referencia al trabajo docente, el siguiente artículo pretende analizar los procesos históricos que vienen configurando las actuales condiciones laborales de los profesores, para después poder reflexionar de forma crítica sobre la reciente ley de Carrera Docente (año 2016) impulsada por el gobierno, mas ampliamente criticada por el gremio docente. La metodología implementada en este artículo consistió en la revisión de documentos legales, artículos y revistas de movimientos docentes.
\end{abstract}

Palabras claves: Neoliberalismo - Trabajo Docente - Educación de Mercado Agobio Laboral.

\section{A PRECARIZAÇÃO DO TRABALHO DOCENTE NO SISTEMA EDUCACIONAL CHILENO}

RESUMO: 0 atual panorama social do Chile é complexo, caracterizado pelas políticas econômicas e sociais baseadas na ideologia neoliberal, implementadas desde os anos '80. 0 anterior sugere que direitos fundamentais como saúde, educação e previdência, sejam abandonados pelo Estado que se manifesta como subsidiário e não como garantidor desses direitos, tendo como objetivo principal o fortalecimento

\footnotetext{
${ }^{1}$ Mestranda em Política Pública e Formação Humana pela UERJ. Possui graduação em Pedagogia, com especialização em Historia pela Pontifícia Universidad Católica de Chile (2007), tem especialização em Infância e Políticas Públicas pela Universidad de Chile (2009), desenvolvendo-se profissionalmente no campo da educação. Email: pssantib@uc.cl

2 Mestre em Educação pela Univerdade Federal Fluminense UFF (2016). Possui graduação em Pedagogia com especialização em matematica pela Pontifícia Universidad Católica de Chile (2007) e, nos últimos dez anos, desenvolve-se profissionalmente na área da educação formal e não formal. Email: gildeyk@gmail.com
} 
do livre mercado em todas as áreas possíveis. Considerando a situação de crise em que se encontra a educação chilena e em referencia ao trabalho docente, o seguinte artigo pretende analisar os processos históricos que configuram as atuais condições laborais dos professores, para poder refletir de forma crítica sobre a recente Lei de Carrera Profesional Docente (ano 2016) aprovado pelo governo, mas amplamente criticada pelo grêmio docente. A metodologia implementada neste artigo consistiu na revisão de documentos legais, artigos e revistas dos movimentos docentes.

Palavras-Chave: Neoliberalismo - Trabalho Docente - Educação de Mercado Carga de Trabalho.

\title{
THE PRECARIOUSNESS OF TEACHING WORKING IN THE CHILEAN EDUCATIONAL SYSTEM
}

\begin{abstract}
Chile's current social scenary is complex, based on the economic and social policies of the neoliberal ideology, implemented in the 1980s. The previous government suggests that fundamental rights such as health, education and social security should be abandoned by the State that manifests itself as a subsidiary and not as a guarantor of these rights. The main objective is to strengthen the free market in all possible areas. Considering the current crisis situation in chilean education, which includes teaching work, the following article intends to analyze the historical processes that has been shaping the current working conditions of teachers, in order to reflect critically on the recent Law of Carrera Profesional (2016) approved by the government, unwhile criticized by the teaching staff. The methodology used in this article consists in the review of legal documents, articles and also magazines of some of the teachers' movements.
\end{abstract}

Keywords: Neoliberalism - Teaching Work - Market Education - Working Time

\section{Introducción}

Las actuales condiciones laborales docentes del sistema educativo chileno responden a un contexto histórico-político que se enmarca en las políticas impulsadas desde la dictadura de A. Pinochet (años 80) y profundizadas con los gobiernos venideros, basadas en la ideología económica neoliberal. Lo anterior generó que derechos fundamentales como salud, educación y pensiones sean abandonados por un Estado que se manifiesta como subsidiario y no como garante de estos, teniendo como objetivo central, fortalecer el libre mercado en todas las áreas posibles.

En este nuevo contexto social del país, el gremio docente es duramente desarticulado, al incorporarse distintos tipos de empleadores y normativas laborales. Ejemplo de esto es la imposición del Colegio de profesores de Chile, creado en el año 1974 durante la dictadura, sustituyendo a la anterior forma de organización docente que era regida por el Sindicato Único de los Trabajadores de la Educación. 
Durante los últimos años, el cuerpo docente se ha visto enfrentado a nuevos decretos y proyectos de leyes que intentan delimitar su formación, desarrollo y labor profesional. Estos temas han abierto debates profundos, volviendo más enérgico al grupo de profesores, generando vertientes ideológicas diversas y nuevas voces que se han levantado a la cabeza de los principales pilares representativos del gremio.

Mas, un hecho importante marca la necesidad de profundizar en esto, nos referimos a la actual Ley de Carrera Profesional Docente promulgada en abril del año $2016 \mathrm{Y}$ a la lucha que se genera por parte de los profesores, organizados en diferentes movimientos sociales, cuando se conoce el proyecto de ésta en 2015.

Así, este artículo pretende reflexionar y analizar la situación laboral docente actual, generando una conexión entre lo que hoy enfrenta el profesorado y las ideologías político-económicas que vienen enmarcando el destino del país desde hace cuatro décadas.

La metodología para este trabajo se dividió en dos etapas: un primer momento de la investigación se efectuó en 2015, mediante una revisión de informes que analizan el estado actual de la educación chilena, las condiciones laborales de los docentes y las actuales legislaciones que han afectado al trabajo docente, principalmente el proyecto de ley de Carrera Docente que se impulsó aquel año. En paralelo, se realizó un seguimiento al movimiento docente que surgió como respuesta a aquel proyecto, a partir de la revisión de sus reivindicaciones a través de documentos como revistas y páginas web publicados por los mismos movimientos. La segunda etapa, se efectuó a comienzos de 2017, con la revisión de la Ley de Carrera Docente $N^{\circ} 20.903$ (la cual fue recientemente promulgada). Es así como se logró una revisión de los cambios entre el proyecto y la ley ya legislada, pudiendo entender en profundidad de qué manera estos cambios acogieron las reivindicaciones del profesorado.

\section{Proceso de neoliberalización en Chile.}

Chile, acoge y orienta sus primeiros pasos hacia la configuración de la ideología neoliberal durante la dictadura cívico-militar (1973-1990), promoviendo un profundo reordenamiento de todas las estructuras jurídicoadministrativas. Fundamenta esta ideología en el crecimiento económico nacional, discurso que se utilizaba para convencer a la ciudadanía de un esplendoroso desarrollo del país: "Uno de los mitos más difundidos del neoliberalismo es que para mantener sostenidamente los ritmos del crecimiento económico es 
necesario reducir la intervención estatal a su mínima expresión" (RUIZ; BOCCARDO, 2015, p. 28).

Por medio de las reformas implementadas a partir del año 1979, conocidas como las siete modernizaciones, se promueven acciones que repercuten a nivel económico y social, como recortes en el gasto público, reforma tributaria, se promueve la liberalización del mercado y la inversión extranjera directa, muchas empresas pertenecientes al Estado pasan a manos de privados y se protegen los derechos de propiedad privada (BIBLIOTECA NACIONAL DIGITAL).

De este modo, y con un evidente cambio en el rol del Estado pasando de ser un Estado garante a un Estado subsidiario de derechos, durante la dictadura muchos servicios e importantes sectores de la actividad económica pasan del Estado a manos de privados (más de 300 empresas públicas fueron subastadas y entregadas a empresarios privados ${ }^{3}$ ); por ejemplo, se modifica y privatiza el sistema previsional, creándose las Administradoras de Fondos de Pensiones (AFP), reforma que "[...] entregan a cierto grupo financiero el control de las cotizaciones de millones de trabajadores" (RUIZ; BOCCARDO, 2015, p. 24); en salud, se reduce considerablemente los presupuestos estatales, abriendo espacios para que privados la ofrezcan como un bien de consumo a comprar en las Instituciones de Salud Previsional (ISAPRES), que prometen ofrecer una calidad muy superior a la abandonada salud pública; en educación, se firma la Ley Orgánica Constitucional del Estado (LOCE) que fija la normativa legal para que la educación sea mercantilizada.

Con el paso de los años y en los gobiernos post dictadura, el Estado mantiene su rol apenas subsidiario de derechos y la agenda neoliberal es legitimada, por medio de políticas que preservan el interés del mercado. Se privatizan recursos naturales, el sector privado toma el control de las actividades mineras y de energía; en materia laboral, continúan restringidas las condiciones para la sindicalización, se desarrolla una agenda de flexibilización laboral que se traduce en precarización e informalización de las condiciones laborales; se comienza a concesionar las obras públicas estatales; se firman tratados de libre comercio que potencian políticas que abren e integran a Chile con los centros del capitalismo mundial (RUIZ; BOCCARDO, 2015).

\footnotetext{
${ }^{3}$ Para un análisis detallado ver: Monckenberg (2001).
} 
Así, los ciudadanos nos vemos enfrentados a una forma de vida que se diferenciará, dependiendo de nuestra capacidad económica, de una mayor o menor calidad en el acceso a estos nuevos servicios (antes derechos).

\section{Neoliberalización de la educación}

El proyecto país que es idealizado por los grupos hegemónicos en dictadura, genera grandes modificaciones al sistema educativo: “[...] la dictadura rompió con toda la concepción republicana de la educación que estaba presente desde la creación misma del Estado Nacional chileno" (GONZÁLEZ, 2015, p. 23) pasando a configurarla como una educación al servicio de un modelo neoliberal.

En una primera etapa, el Estado deja de tener un rol garante y pasa a ser subsidiario, interviniendo sólo cuando el sector privado no lo hace. Para González (2015), esto es posible dadas tres condiciones fundamentales presentes en la Constitución Política de la República de Chile (1980) y en la reforma al sistema primario y secundário de enseñanza por medio de la Ley Orgánica Constitucional de Enseñanza (LOCE- 1990): i) Fundamento jurídico; el derecho a la educación no está sujeto al amparo del Recurso de Protección en los tribunales de justicia ${ }^{4}$. La responsabilidad recae en los padres, madres y/o apoderados y el rol del Estado es de promotor de este derecho. (ii) Financiamiento (Ley de subvenciones 1981); se eliminan los presupuestos fijos estatales y se asigna una subvención a la demanda o vouchers acorde a cada estudiante matriculado. Además, cada municipio podrá otorgar un financiamiento extra acorde a sus posibilidades. Este sistema genera grandes diferencias entre escuelas, por ejemplo; escuelas municipales de baja y alta matrícula, escuelas de municipios con mayor y menor recursos, escuelas rurales y urbanas. (iii) Administración; la llamada Municipalización se inicia en el año 1979 facultando el traspaso de los establecimientos educacionales estatales desde el Ministerio de Educación a los municipios. Este cambio incorpora también el ingreso de privados en la figura de sostenedores de escuelas que recibirán subvención estatal. Así, las escuelas quedan divididas en: a) Escuelas Privadas; de financiamiento y administración privada, b) Escuelas Municipales; con aporte estatal, pero con administración municipal y c) Escuelas Particulares subvencionadas; con aporte estatal y administración privada.

\footnotetext{
${ }^{4}$ A diferencia del derecho a la propiedad, la libertad de enseñanza, el derecho a adquirir dominio sobre toda clase de bienes, o desarrollar cualquier actividad económica, que sí son amparados por el Estado en su lógica neoliberal.
} 
Todo lo anterior se basa en la ideología que sustenta la educación de mercado en Chile, recordándonos las palabras de Milton Friedman' ${ }^{5}$, Luis González intenta develar el origen que da base a nuestras políticas educativas, y al resto de ella también: "[...] una sociedad donde el mercado inunde y regule todas y cada una de las relaciones humanas" (GONZÁLEZ, 2015, p. 50). Así, la educación es entendida como "un medio para alcanzar un fin superior y último, esto es, la sociedad de mercado y el despliegue del individuo económico. Es precisamente esta racionalidad instrumental el sostén filosófico-ideológico del modelo educacional chileno" (GONZÁLEZ, 2015, p. 51). Es este pensamiento el que predomina ideológica, administrativa y legalmente en el país.

Según diversos estudios, en la actualidad, la educación chilena se caracteriza por: a) Tanto en su forma administrativa como de financiamiento, predomina el sector privado; b) Es un sistema que genera alta segregación y desigualdad social.

En relación a lo primero, podemos revisar los resultados del estudio Education at a Glance (OECD, 2014):

- Chile es el país con menor matrícula escolar en escuelas públicas en Latino América; sólo un 37,5\% de las matriculas son ofrecidas por el sistema público. El promedio internacional de los países pertenecientes a la OCDE es de $82 \%$. Además, es el tercer sistema educativo en el mundo con la menor oferta pública estatal en educación.

- Si bien el gasto público en educación sigue aumentando, la financiación privada sigue dominando en todos los niveles del sistema educativo; esta inversión privada tiene como principal financista a las familias de estos estudiantes y predomina en el sector terciario (educación superior).

- Chile tiene la menor proporción del gasto público en la educación superior de todos los países de la OCDE y, consiguientemente, la mayor proporción en gasto privado, la que en Chile llega al 76\%, mientras que el promedio de la OCDE es de apenas 31\%.

En cuanto a la segregación y desigualdad social, podemos decir que pese a existir buenos índices de cobertura escolar y acceso a la educación en el país - en el año 2013 alcanza un 90,2\% en cuanto a Tasa Neta de Escolaridad (TNE) (CHILE,

\footnotetext{
${ }^{5}$ Economista y estadista de la Universidad de Chicago. Bajo sus ideologías neoliberales, los economistas chilenos plantearán las reformas del país desde los años 1970 en adelante.
} 
2014)- esto no se relaciona con buenos resultados en cuanto a calidad y equidad del sistema educativo. En el informe 7 fenómenos sobre educación y desigualdadCIES se plantea que en Chile, la variable nivel socioeconómico de las familias es la que más influye en los resultados educativos, es decir, la estructura de oportunidades está determinada socialmente a partir de la clase social de pertenencia (MAYOL et alli, [2011?]). Lo anterior es refrendado por un informe publicado por la Organización para la Cooperación y el Desarrollo EconómicosOCDE en conjunto con el Ministerio de Educación de Chile -MINEDUC, en el cual denuncian que "el sistema educativo chileno está conscientemente estructurado por clases sociales" (OCDE, 2004, p. 277).

Elacqua y Santos (2013), analizando la matricula del año 2008 en las escuelas de Chile, demuestran cómo la pertenencia a cada escuela está directamente ligada a la situación económica del estudiante. Lo anterior, significa que al interior de cada escuela sólo es posible encontrar una homogeneidad de clase, es decir, que los pobres estudian con los pobres y los ricos con los ricos.

En el caso de la educación superior, las universidades, de fuerte carácter público hasta antes de 1973, se vieron coartadas en su actuar; se disgregan las sedes regionales de las instituciones mayores, quema masiva de bibliotecas, desaparecimientos y asesinatos de profesores y estudiantes, cierre de facultades del área social de algunas universidades. Adicionalmente, el régimen impone una nueva forma de concebir la educación superior, mercantilizada y despolitizada, impulsando en el año 1981 la Ley General de Universidades, que fomenta y otorga las licencias para crear universidades privadas. Se crea la Ley de Financiamiento, que obliga a las universidades públicas a competir con los planteles privados para recibir financiamiento estatal a través del Aporte Fiscal Indirecto (AFI) ${ }^{6}$ y se les obliga a autofinanciarse. Con esta nueva necesidad, las universidades comienzan a cobrar matrículas y aranceles a los estudiantes, ofreciendo créditos para financiar los estudios de los estudiantes más pobres (RUIZ; BOCCARDO, 2015).

Pese a una serie de reformas, el Estado chileno continúa asumiendo un rol subsidiario al momento de abordar los derechos fundamentales. Aún no existe garantía constitucional de protección por parte del Estado en temas educativos, la educación sigue siendo dirigida como un producto más del mercado.

\footnotetext{
${ }^{6}$ Subvención otorgada a las instituciones que matriculen a los estudiantes de mejor puntaje en la prueba única de selección universitaria.
} 


\section{Trabajo Docente}

\section{Trayectoria desde la dictadura hasta el 2016}

Durante la dictadura el gremio docente es duramente desarticulado tanto en su ámbito organizativo propiamente tal, como en sus relaciones laborales con el conjunto del profesorado, al incorporarse distintos tipos de empleadores y normativas laborales. Así, para la desarticulación organizativa el régimen implantó la sustitución del Sindicato Único de los Trabajadores de la Educación por el Colegio de Profesores de Chile en 1974, cuya afiliación tuvo carácter obligatorio y sus dirigentes eran designados por el régimen militar.

La fragmentación de los profesores en cuanto a sus relaciones laborales, fue implantada mediante la Ley de municipalización y a partir de la incorporación de colegios particulares subvencionados. Con la municipalización se impulsa la descentralización de la educación con el traspaso de las escuelas públicas, de enseñanza primaria y secundaria, desde el Estado central a cargo del Ministerio de Educación hacia los municipios. Con esta medida los profesores dejan de ser funcionarios del Estado para ser empleados de las municipalidades, con lo que pierden derechos históricos: como estabilidad laboral, homogeneidad de salario y valor hacia la experiencia y antigüedad (NÚÑEZ, 1999), lo más grave es que al ser traspasados a las municipalidades serán sometidos a un régimen laboral y previsional propio del sector privado (NUÑEZ et al., 1984). También se produce una gran segregación del profesorado municipal, ya que éstos tendrán tantos empleadores como municipalidades existan, las cuales implementarán distintas decisiones y normativas para sus trabajadores. Situación que se ve aún más mermada, con la introducción de colegios subvencionados, pues el profesorado adquirió aún más tipos de empleadores y regulaciones laborales, lo que conduce a una grave dificultad para estructurarse en una sola organización nacional (GONZÁLEZ, 2015).

Observamos entonces que en la década de los ochenta, debido a la nueva forma de administrar los establecimientos educativos, los profesores de instituciones estatales pasan a ser regidos por las leyes laborales de los trabajadores del sector privado, disminuyendo considerablemente los derechos que habían obtenido a lo largo de la historia (RUIZ; BOCCARDO, 2015).

Después de la llegada de la democracia, en 1991 se decretó la Ley Nº 19.070 que instauró un Estatuto de los Profesionales de la Educación, que tuvo como misión principal mejorar las condiciones paupérrimas que alcanzó el trabajo 
docente durante la dictadura, especialmente en materia de salario. Para esto, se creó la Remuneración Básica Nacional, pero el mayor porcentaje del salario docente mejoró mediante bonos, generando remuneraciones fuertemente heterogéneas dentro del gremio.

A pesar de las promesas de mejoramiento de las condiciones del trabajo docente, este Estatuto conserva el régimen municipal, por lo que los docentes no pudieron recobrar su estatus de funcionarios públicos, como tampoco la totalidad de derechos que gozaban antes de la dictadura.

Otro problema que se aloja en este decreto, es que promueve una vez más la ramificación del profesorado, pues en su título IV se establece que los profesores que se desempeñen en colegios particulares pagados y subvencionados serán regidos por el Código del Trabajo en todo aquello que no esté expresado en dicho título, mientras que sus colegas municipales lo harán por el estatuto en su cabalidad (CHILE, 1991). Con esto se intenta producir división dentro del gremio, ya que para igual trabajo se desenvuelven diferentes marcos legales según la dependencia administrativa del colegio en donde trabaje cada profesor (GONZÁLEZ, 2015).

Desde 1995, se introdujeron modificaciones al estatuto docente que flexibilizaron aún más el trabajo de los profesores: se autorizó el despido de docentes municipales por requerimientos del servicio ${ }^{7}$. Se ajustó la norma que permite contratar profesores a plazo fijo dentro del sistema municipal, ampliándola hasta el $20 \%$ de las horas de dotación docente, lo cual, produjo flexibilidad laboral para los profesores municipales. Se introdujo el principio de incentivo por desempeño, tanto colectivo, como individual, el cual consiste en un incentivo remuneracional selectivo para la planta docente de los establecimientos que sean declarados como destacados, además de un incentivo extra para los docentes de dichos establecimientos que también sean señalados como destacados. Por último, se instaura el Sistema Nacional de Evaluación Docente, el cual ha fortalecido la óptica punitiva y de desconfianza hacia el magisterio, profundizando el incentivo individual monetario, mediante la Asignación Variable de Desempeño Individual (GONZÁLEZ, 2015).

\footnotetext{
${ }^{7}$ Con la Ley 19410 promulgada en 1995 se introdujeron modificaciones a la Ley N 19.070. Entre éstas se incorporaron nuevas razones de despido de docentes del sector municipal, estas razones son: i. Variación en el número de alumnos del sector municipal de una comuna; ii. Modificaciones curriculares; iii. Cambios en el tipo de educación que se imparte; iv. Fusión de establecimientos educacionales; v. Reorganización de la entidad de administración educacional (CHILE, 1995).
} 
En síntesis, vemos que el estatuto docente sólo subsanó las condiciones paupérrimas que alcanzó el trabajo docente durante la dictadura, en cuanto a salario y algunos derechos. Sin embargo, esta ley no realizó cambios drásticos con respecto al trabajo docente y a la educación que se estableció en dictadura. Por el contrario, este estatuto ha profundizado el modelo neoliberal impuesto mediante políticas de segregación hacia el gremio, flexibilización laboral y la introducción de asignaciones por mérito individual.

\section{Condiciones laborales de los docentes en el sistema educativo chileno}

Para analizar las actuales condiciones laborales de los docentes en Chile revisaremos un estudio realizado por United Nations Educational, Scientific and Cultural Organization -UNESCO ${ }^{8}$ en el año 2005, sobre condiciones de trabajo y salud docente en Chile, el cual plantea los siguientes resultados (PARRA, 2005):

Turnos de trabajo y descanso: Un tercio de los encuestados trabaja en dos o tres turnos durante el día y un 19,7\% tiene un trabajo adicional a la docencia. Se observa que existe una gran cantidad de tareas docentes que son realizadas fuera del horario laboral; el $50 \%$ de los encuestados realiza seis o más quehaceres de su trabajo fuera del horario asignado para éste. A esto se suma, la ausencia de descanso adecuado; un 45\% aprox. de los profesores destina menos de 15 minutos al descanso durante la jornada laboral.

Condiciones materiales de trabajo: los establecimientos visitados, en su mayoría, se encuentran en buenas condiciones. Sin embargo, espacios como los baños, comedores, salas para el descanso y oficinas para preparación de clases, no se encuentran en buen estado, no son adecuados o no son suficientes para el profesorado. Las pizarras en general están en buen estado, pero las sillas y mesas de los profesores no son adecuadas para éstos. Los puntos más críticos son la ausencia de espacios para educación física e insuficiencia de equipamiento para disciplinas artísticas.

Exigencias ergonómicas del trabajo: En cuanto a la medición de los niveles de exigencia ergonómica, la gran mayoría de los profesores encuestados se ubica en los tramos alto y muy alto, medido según la cantidad de factores ergonómicos que interfieren en su trabajo. Los tres factores que obtuvieron mayor frecuencia en los

\footnotetext{
${ }^{8}$ Estudio de muestra realizado a 158 docentes de ambos sexos, pertenecientes a colegios de enseñanza básica y media de administración municipal de Santiago de Chile.
} 
docentes encuestados son: trabajar en ambiente ruidoso (48.7\%), forzar la voz (47.1\%) y estar de pie toda la jornada laboral (35.9\%).

Condiciones sociales de trabajo: para los docentes encuestados las relaciones entre colegas es más satisfactoria que con las autoridades de los colegios. Los docentes sostienen que los superiores no promueven un ambiente adecuado de trabajo, pues perciben que la supervisión que éstos realizan no busca ser un real apoyo hacia el trabajo.

Los docentes encuestados expresaron sentirse más exigidos por las condiciones en las que se realiza el proceso educativo, que por las características propias de los estudiantes. De las variables sobre los alumnos, las expresadas con mayor frecuencia son: comportamiento indisciplinado del grupo, problemas de aprendizaje y repitencia. Y las variables con mayor frecuencia sobre el proceso fueron: excesivo número de alumnos por sala, falta de cooperación por parte de padres o tutores y ausencia de apoyo pedagógico de especialistas en la escuela.

Problemas de salud: La principal enfermedad diagnosticada por los médicos a lo largo de la carrera docente es la disfonía o afonía con un $46 \%$, seguida de enfermedades relacionadas con el control de la ansiedad: colon irritable con un $44 \%$ y estrés con el $41 \%$. Además del historial de enfermedades diagnosticadas, se les preguntó a los profesores por los malestares sufridos con frecuencia durante el último año, de esta forma se construyó el siguiente perfil acumulativo de problemas de salud: i) Patologías de carácter crónico; con presencia de enfermedades como hipertensión arterial (asociado al perfil demográfico, ya que la mayoría de los encuestados es de edad avanzada). ii) Salud mental; se presentan problemas psicosomáticos, estrés, depresión, angustia e insomnio. iii) Problemas asociados a exigencias ergonómicas; con enfermedades como disfonía, problemas musculo esqueléticos y várices.

Por otra parte, según datos que nos muestra OECD (2015) la proporción de estudiantes por cada docente en Chile es mayor que el promedio OCDE, con 23 estudiantes por profesor en enseñanza básica y de 25 en enseñanza media (promedio que es superado solo por México), mientras que esta cifra para el resto de los países de la OCDE es de 15 y 13 estudiantes respectivamente. Es importante considerar que este dato es solo un promedio, pues la normativa vigente expresa que el máximo de alumnos por sala puede llegar a 45 para enseñanza parvularia, básica y media.

De esta forma, observamos que el trabajo docente en Chile es altamente exigente, pues en general los profesores están sometidos a enfermedades 
asociadas con el quehacer docente: ausencia de descanso dentro de la jornada laboral, malas condiciones materiales para el trabajo, alta exigencia ergonómica, exceso de trabajo que no alcanza a efectuarse durante la jornada laboral y se termina realizando en casa obstruyendo el tiempo libre de cada docente.

\section{Carrera Profesional Docente}

Las condiciones laborales de los docentes, que fueron descritas en el apartado anterior, junto a la formación inicial que éstos reciben en las Universidades e Institutos Profesionales que imparten pedagogías, han sido tema de debate en los últimos años. Si bien se estrena una reciente legislación de Carrera Docente, el debate sigue y se ha vuelto aún más enérgico.

Contexto previo a la promulgación de la ley de Carrera Profesional Docente.

El día 20 de abril de 2015 la actual Presidenta Michelle Bachelet, por medio del mensaje $\mathrm{N}^{\circ}$ 165/363(CHILE, 2015a), presenta ante la Cámara de Diputados un proyecto de ley cuyo objetivo es proponer la creación de un sistema profesional docente, en el marco de la Reforma Educacional que está siendo propuesta por medio de diversos proyectos de leyes, entre estos el proyecto Sistema de Desarrollo Profesional, conocida como Carrera Profesional Docente (en adelante la llamaremos CPD). En este mensaje se explicita que esta propuesta fue creada bajo una dinámica participativa y dialogal con diversos actores como el Colegio de Profesores de Chile A.G, la sociedad civil y una iniciativa llamada Plan Maestro y que junto a las propuestas de cada uno de estos se creó la llamada CPD.

Sin embargo, luego de hacerse público el documento y las políticas a implementar, el rechazo desde los docentes fue profundo. El día 11 de mayo se realizó una consulta nacional por parte del Colegio de Profesores de Chile en que un $97 \%$ de los docentes se manifiesta en contra de esta propuesta, demostrando que el diálogo y creación participativa del proyecto al que se refiere la presidenta en su mensaje no reflejaba el parecer de los profesores.

Los diálogos con los docentes a los que se refiere el Gobierno fueron con 20 mil profesores y profesoras, lo que representa tan solo un $10 \%$ del profesorado a nivel nacional, a través de actividades fuera del año lectivo y dos jornadas de información que dependían de la voluntad de cada sostenedor de escuelas subvencionadas. Además, las instancias de diálogo propuestas por el Gobierno 
fueron consultivas y no resolutivas. De esta forma no se llegó a ninguna especie de acuerdo, ni de consenso. Por lo que podemos sostener que el proyecto de ley se realizó sin el profesorado (LAMA, 2015).

Bajo este escenario el 1 de junio comenzó un inédito paro indefinido por parte del profesorado en la mayoría de los colegios municipales a lo largo de todo Chile, cuya exigencia fue el retiro inmediato del proyecto de ley de CPD. Tras 58 días de suspensión de clases y movilizaciones, el paro arrojó como resultado; un fuerte compromiso del profesorado proveniente de colegios particulares subvencionados a través de actividades que no implicaron necesariamente suspender su trabajo; una gran adhesión ciudadana hacia las demandas del profesorado; cambio de ministro de educación y la concreción de dos mesas de trabajo tripartitas compuestas por miembros del Ministerio de Educación, diputados pertenecientes a la comisión de educación y por el Colegio de Profesores de Chile, en donde luego de discutir sobre el proyecto de ley, se formularon algunos cambios al proyecto.

A pesar de las movilizaciones, el 23 de julio del mismo año se aprobó en la Cámara de Diputados la idea de legislar sobre el proyecto de ley de CPD, tras lo cual el Colegio de Profesores volvió a realizar una consulta nacional a sus bases para saber el nuevo destino de las movilizaciones y del paro, los resultados de ésta fueron: contabilizados más de 19 mil votos, el 64\% de los docentes estuvo de acuerdo con el fin de la paralización, mientras que el 92 \% siguió rechazando la iniciativa impulsada desde el Gobierno. De esta forma la última semana de julio el paro docente concluyó con consecuencias de descuentos y hasta despidos para algunos de los docentes movilizados.

\section{Ley de Carrera Profesional Docente}

En abril de 2016, bajo el slogan "La Pedagogía Necesita a los Mejores" se promulgó la Ley $\mathrm{N}^{\circ} 20.903$ (CHILE, 2016), la cual establece al Sistema de Desarrollo Profesional Docente, y expresa tener como objetivo el dignificar la docencia, apoyar su desempeño y aumentar su valoración. Este sistema define el trabajo para educadores y educadoras de párvulo, educadores y educadoras diferenciales, y para profesores de enseñanza básica y media que se desempeñen en establecimientos municipales o subvencionados, y se estructura mediante los siguientes pilares: Formación Inicial, Ingreso a la Carrera, Progresión en la Carrera Docente, Formación en servicio y Distribución de horas lectivas y no lectivas. 
Es importante mencionar que el inicial proyecto de CDP sufrió cambios hasta convertirse en la actual ley, los cuales tomaron en cuenta las críticas que planteó el fuerte movimiento docente. Entre estos se encuentra la incorporación de los profesionales de párvulo y educación diferencial, la incorporación a la carrera de los profesionales recién egresados sin ninguna experiencia laboral, que en el proyecto originario tenían que esperar a certificarse para recién poder entrar.

Para efectos de este artículo sólo nos basaremos en explicar y analizar lo que estipula la ley para el trabajo de profesores en enseñanza básica y media. A continuación, se presentan los principales pilares de la CPD y en paralelo, las principales críticas que se le hacen por parte del profesorado organizados en diferentes movimientos sociales ${ }^{9}$ :

a) Asegurar la calidad de la formación inicial docente: Establece que sólo las universidades acreditadas y cuyos programas de Pedagogía estén acreditados por el Ministerio de Educación podrán impartir la formación de profesores; plantea nuevos y más estrictos requisitos para seleccionar a los futuros estudiantes de pedagogía; las universidades deberán realizar obligatoriamente dos evaluaciones diagnósticas (al inicio y al término de la formación); crea modelos de capacitación temprana de jóvenes con intereses y talentos pedagógicos.

Críticas: En este punto fueron varios los aportes que se consideraron de las demandas que el profesorado realizó al inicial proyecto de ley de 2015, como por ejemplo, establecer criterios por ley para la acreditación de las universidades y que la evaluación diagnóstica no sólo servirá como sustento de información a las universidades, sino que también se verán en la obligación de generar procesos de retroalimentación y deberán tomar medidas para apaliar los posibles bajos resultados. Sin embargo, la evaluación de la formación universitaria en pedagogía podría llevar a que las instituciones acomoden sus prácticas educativas a los criterios evaluados en esta prueba, lo que podría generar una estandarización curricular en la formación de profesores debido al modelo educativo mercantil y competitivo que existe también en la educación terciaria del país.

Por otra parte, los estándares para la formación inicial dan cuenta de un profesor sin autonomía, capacidad crítica y reflexión, además de haber sido creados sin un proceso participativo que involucre a la comunidad educativa.

\footnotetext{
${ }^{9}$ Para conocer y analizar las principales críticas que se le realizan a la Ley de CPD se han tomado los aportes de varias organizaciones - MUD, Acción Docente, Vamos Construyendo, Centro de Alerta, entre otros- a las cuales pudimos acceder a través de los siguientes documentos: CAMPAÑA POR UNA NUEVA EDUCACIÓN, 2015; GÓNZÁLEZ, 2016; LAMA, 2015; MUD, 2016; OPECH, 2015.
} 
b) Apoyar la inserción laboral de los profesionales de la educación: Proceso de inducción a los profesionales docentes que se inician en el aula que busca acompañar la construcción temprana de la profesionalización docente e incidir en la disminución del porcentaje de deserción del sistema escolar de los profesores iniciantes. Las características de esta inducción son; acompañamiento durante el primer año de ejercicio docente; inscripción voluntaria; cantidad limitada de cupos; sólo pueden participar quienes estén contratados; el apoyo será realizado por un docente mentor perteneciente a la misma comuna y nivel o área de trabajo.

Críticas: La inducción debe ser universal y no dependiente de cupos; el mentor debe ser un docente de aula en ejercicio, privilegiando a los que trabajan en el mismo establecimiento o en una misma realidad educativa.

c) Aumento de las horas no lectivas: Hasta el año 2016 los docentes disponían de un $25 \%$ de sus horas de trabajo para organizarse fuera del aula (horas no lectivas), y el resto del tiempo de sus horas de contrato (75\%) correspondía a horas dentro del aula. Con la Ley se establece un aumento gradual de las horas no lectivas hasta llegar a un 35\% de sus horas de trabajo en 2019.

Críticas: No se considera la demanda de 50-50 que se viene realizando desde hace algunos años por los docentes ${ }^{10}$ sino que se ofrece aumentar gradualmente hasta llegar en el año 2019 a un 35\% de horas no lectivas por contrato, lo que en la práctica implica un cambio mínimo a las condiciones laborales. Además, se suma que en la ley se establece que este aumento de horas no lectivas sólo se realizará bajo auspiciosas condiciones económicas del país, pudiendo generar estancamientos de la puesta en marcha de este beneficio.

d) Progresión en la Carrera Docente: A partir de esta legislación la carrera de los docentes estará estructurada en tramos. El avance entre un tramo a otro se efectuará a través de un proceso de certificación que se obtiene de una evaluación que posee dos instrumentos: evaluación de conocimientos específicos y pedagógicos y la elaboración de un portafolio de competencias pedagógicas.

Los tramos por los que debe avanzar cada profesor se dividen en obligatorios: inicial, temprano y avanzado; y voluntarios: experto I y experto II. Para acceder a cada tramo es necesario contar con años de experiencia según se vaya avanzando en la carrera; así, para acceder al tramo profesional temprano y avanzado es necesario poseer 4 años de experiencia como mínimo, ocho para el experto I y doce para el experto II. Los tramos están creados para los siguientes perfiles

\footnotetext{
${ }^{10}$ Para mayor información se recomienda visitar el sitio http://porladignidaddocente.blogspot.com.br/
} 
profesionales: Inicial; para quienes ingresan al sistema laboral docente sólo contando con su título profesional. Temprano; en este tramo se espera que el docente avance hacia la consolidación de su experiencia y competencia profesional. Avanzado; en este tramo el docente ha logrado el nivel esperado de consolidación de sus competencias, demostrando capacidad para lograr aprendizajes en la totalidad de sus estudiantes, de acuerdo a las necesidades de cada uno. Experto I; tramo de carácter voluntario. Pensado para docentes que se encuentren en el tramo avanzando por lo menos cuatro años, y que tengan un desempeño sobresaliente. Los docentes que estén en este tramo tendrán acceso preferente a funciones de acompañamiento y liderazgo pedagógico. Experto II; tramo de carácter voluntario. Pensado para docentes que se encuentren en el tramo Experto I por lo menos cuatro años, y que posean un desempeño de excelencia. Al igual que en el anterior, tendrán acceso preferente a funciones de acompañamiento y liderazgo pedagógico.

La certificación, consiste en rendir satisfactoriamente los dos instrumentos evaluativos ya mencionados, que se aplicarán cada cuatro años. Sólo de alcanzar un alto nivel en la evaluación de conocimientos específicos y pedagógicos y en el portafolio podrán no rendir estos instrumentos en el proceso de certificación siguiente. Esta certificación es obligatoria para profesionales de la educación que se encuentren en los tramos inicial y temprano, mientras que para los que se encuentren en los tramos avanzado, experto I y II serán voluntaria.

Los docentes que se encuentren en el tramo inicial, tendrán sólo dos oportunidades para avanzar al tramo temprano ${ }^{11}$, de no lograrlo serán desvinculados del sistema. Los docentes que ingresen al nuevo sistema desde el año 2025 tendrán también solo dos procesos de reconocimiento para avanzar desde el tramo temprano al avanzado, de no lograrlo, después de dos años tendrán que volver al tramo inicial, pero con la obligación de avanzar al tramo avanzado en un plazo de dos años, en el caso de que no obtengan los resultados esperados serán desvinculado del sistema ${ }^{12}$, sin poder ser contratado por el mismo u otro centro educativo municipal o subvencionado. Por otra parte, los profesionales que hayan ingresado a los tramos temprano, avanzado, experto I y II no retrocederán a tramos anteriores de la carrera docente.

\footnotetext{
${ }^{11}$ La Ley contempla que reciban apoyo formativo en este proceso.

12 Estos docentes habrán ingresado a estudiar pedagogía bajo los nuevos estándares de calidad que establece el sistema dentro de esta misma Ley.
} 
Cada tramo involucra un bono en la remuneración, la cual es más considerable en la medida que se avanza en la carrera. Además, la ley en su artículo 19 B, menciona que el avance de un tramo a otro significará además asumir crecientes responsabilidades en el establecimiento educacional donde se ejerce.

Críticas: Sin duda este es el punto que implica mayor divergencia entre los elaboradores de la ley y quienes serán sometidos a ella.

Al ser una certificación de carácter individual se enmarca sólo en el sujeto, el cual tiene el riesgo incluso de perder la posibilidad de ejercer su profesión al ser mal evaluado, no contemplando la responsabilidad de la institución que lo formó. También esta individualización promueve la competencia entre colegas y dificulta el trabajo colaborativo por lo que se desecha la oportunidad de potenciar el carácter comunitario de los procesos de aprendizajes y logro de objetivos, ya que cada profesor deberá velar por su desarrollo profesional potenciando el individualismo dentro de una lógica meritocrática. Lo anterior, como todo sistema basado en la meritocracia, considera que los docentes de diversas realidades laborales serán sometidos a un instrumento estandarizado de evaluación, que no considera las complejidades propias de cada contexto.

El proceso evaluativo obliga a trabajar fuera del horario de contrato sin compensación alguna, lo que aumenta el agobio laboral. No genera mayor estabilidad laboral, pues avanzar de tramo no implica contrato indefinido de trabajo. Por el contrario, la inestabilidad laboral aumenta de tal forma que involucra pérdida de derechos ganados históricamente por el gremio docente. Nos referimos al derecho de contrato de trabajo indefinido tras dos contrataciones anuales, pues en el caso de profesores del tramo inicial la inestabilidad e incertidumbre laboral puede llegar hasta 4 años.

Las bonificaciones están pensadas en montos máximos para profesores de 44 horas de contrato y que posean 15 bienios, situación de la que no se beneficia la mayoría de los docentes que es contratado por menos horas y no posee tal antigüedad. Se elimina la Asignación por trabajo en Condiciones Difíciles, la cual es reemplazada por la Asignación de Reconocimiento por Docencia en Establecimientos de Alta Concentración de Alumnos Prioritarios ${ }^{13}$ la cual es supeditada al tramo en el que se encuentre el docente. Si bien los salarios se ven aumentados debido a un incremento en el valor del bono por título y mención (BRP), la Renta Básica Mínima Nacional no se modifica (remuneración por horas

\footnotetext{
${ }^{13}$ El Ministerio de Educación entiende por alumnos prioritarios a aquellos estudiantes que se encuentran en una situación socioeconómica que pueda dificultar su proceso educativo.
} 
de contrato) lo que implica que el sueldo base (sin añadir las bonificaciones) de los docentes continúe siendo muy bajo en comparación con otros profesionales, dificultando el acceso en su vida cotidiana, por ejemplo, para postular a créditos de vivienda o educación para sus hijos.

Este proceso de certificación se suma a las muchas otras evaluaciones que ya se aplican a los docentes (pruebas estandarizadas a sus estudiantes, prueba de ingreso único para acceder a las universidades, evaluaciones locales), sin embargo, ninguna de ellas especifica de qué forma ayuda a mejorar la labor docente o cómo se realizará la retroalimentación de estos procesos evaluativos con los profesores.

Por otro lado, la asignación por tramos, además de poner un tope final de remuneraciones, obliga a los docentes a mantenerse durante años en un mismo nivel de remuneraciones, sin considerar sus potencialidades para avanzar a un nivel mayor de desarrollo docente.

e) Formación en servicio: la ley establece que los docentes puedan contar con el acceso a formación continua gratuita a lo largo de su carrera profesional con el objetivo de brindar un apoyo a los profesores en la progresión dentro del sistema de desarrollo profesional. Para esto el Centro de Perfeccionamiento, Experimentación e Investigaciones Pedagógicas (en adelante CPEIP) del Ministerio de Educación ofrecerá a los profesores cursos, programas y actividades o los coordinará a través de universidades o instituciones (públicas o privadas) acreditadas y sin fines de lucro. Esta oferta de cursos se dispondrá según las necesidades presentes en los docentes y los establecimientos. Es importante mencionar, que estos cursos no comprenden estudios de postgrado.

Además, se establece que en cada establecimiento educacional se realicen planes de formación para el desarrollo profesional, los cuales podrán ser financionados con la Subvención Escolar Preferencial ${ }^{14}$.

Estos cursos, están pensados como un apoyo para avanzar en la carrera docente, pero en ningún caso aprobar en un curso significará avanzar hacia un tramo más avanzado, de hecho la ley en su artículo $\mathrm{N}^{\circ} 12$ expresa que es responsabilidad del profesor el avance dentro de su desarrollo profesional. Por otra parte, cada uno de estos cursos tendrá cupos limitados, por lo que no todos los profesionales de la educación podrán acceder a ellos cada año, por esto

\footnotetext{
${ }^{14}$ La Subvención Escolar Preferencial (SEP) es un beneficio en dinero entregado por el Ministerio de Educación a los sostenedores de escuelas subvencionadas por cada alumno considerado vulnerable socioeconómicamente. Más información en MINEDUC (2017).
} 
tendrán prioridad los docentes que se desempeñen en establecimientos con mayores necesidades según sus estudiantes y por la mayor relación entre la función que cumple el profesional y los contenidos del curso al que postula.

Críticas: La asignación por tramo de desarrollo profesional elimina la asignación por perfeccionamiento que existía para aquellos docentes que han continuado estudios realizando cursos, diplomados o postgrado.

Se observa con recelo que instituciones privadas puedan brindar parte de esta formación continua, ya que a pesar de que se expresa que éstas deberán ser sin fines de lucro, esta práctica se puede convertir en un negocio para los privados con recursos del Estado. Esta desconfianza se sustenta en pasados casos de universidades que en teoría no perseguían fines de lucro, pero que en la realidad estaban envueltas en obtención de ganancias ilícitas.

\section{Conclusión}

El magisterio ha sufrido una serie de continuos dispositivos perpetrados por el Estado para dominarlos. Sin embargo, es el mismo Estado quien entrega a gran parte del profesorado a manos de sostenedores privados; de hecho, en el año 2014 los profesores que se desempeñaba en el sector municipal eran 94.247, mientras que los profesores del subvencionado correspondían a 99.349 (CHILE, 2015b). Lo anterior, podría parecer una contradicción, sin embargo, en la actual sociedad neoliberal regulada por el mercado no lo es, pues el real dispositivo de dominio que se observa en la trayectoria de políticas educativas y de trabajo docente desde la dictadura hasta la actualidad, es la búsqueda de la fragmentación del gremio.

Tal situación de segregación continua con la actual Ley de CPD, pues a pesar de que tanto profesores de colegios municipales (administración municipal), como profesores de colegios subvencionados (administración privada) son exigidos de la misma forma por esta nueva normativa. Los profesionales del sector subvencionado seguirán siendo regidos también por el Código del Trabajo, por lo que se les niegan derechos adquiridos que poseen sus colegas municipales.

Para analizar la reciente ley de CPD quisiéramos comenzar con el slogan de ésta; "La pedagogía necesita a los mejores", pues nos parece que lo que se esconde bajo esta legislación no es generar más dignidad hacia el profesorado como ésta plantea entre sus objetivos, sino que, por el contrario, se propone actuar como un dispositivo que primero clasifique, para después excluir a los docentes que no le 
sirven al sistema, es decir, a los profesores que no estén moldeados como se espera.

Observamos que una de las consecuencias de esta ley es la introducción, o mejor dicho la profundización, de políticas de gerencialismo llevadas a la educación. Éstas tienen por objetivo llevar las lógicas del mundo empresarial a la escuela, lo que se ve plasmado en la búsqueda de la eficiencia y productividad. Así vemos que el gerencialismo educativo realiza grandes exigencias a los profesores, pues ve en ellos un importante recurso educativo. Para esto los somete a evaluaciones permanentes con respecto a sus competencias, con el objetivo de controlar su trabajo, y poder clasificarlos según estándares de calidad y así poder remunerarlos por productividad (SHIROMA, 2004).

Es así como se explican las certificaciones y los bonos por tramo, con los cuales se potencia el individualismo y la competencia. De tal modo, surgen nuevas relaciones de poder, pues el sistema ahora se presenta más vertical incluso, incorporando nuevas formas y agentes de control para los profesores. Control que está sustentado en la inestabilidad laboral que profundiza esta nueva legislación, tal como plantea Sennett (2000), la evaluación constante y el riesgo de pasar de una categoría a otra, es decir, el riego de tener éxito o fracaso produce en los trabajadores sensaciones de inestabilidad, confusión y vulnerabilidad laboral. Por esto, con la CDP los profesores se encuentran constantemente en riesgo, por lo tanto, constante vulnerables.

Este control hacia el profesorado lo percibimos también en la incorporación de la formación continua, pues como estos cursos serán administrados por el CPEIP los contenidos de éstos estarán acordes a lo que busca el Estado para el quehacer docente y no a las reales inquietudes intelectuales de los profesores. Por ejemplo, vemos que sería muy extraño encontrar cursos de pedagogía que posean una corriente crítica. Esto queda en evidencia al dejar fuera la formación en estudios de postgrado, que permiten la reflexión profunda de temáticas vinculadas a la labor docente.

Con el estatuto docente, las remuneraciones subieron, pero principalmente en base a asignaciones, pues no se buscaba un alza del sueldo base del conjunto del profesorado. Medida que se expresa aún más con el actual la actual Ley, a partir de las asignaciones por tramo las remuneraciones de los profesores serán cada vez más heterogéneas, pues responderán a una multitud de variables, como bonos por tramos (mérito individual) acorde a las horas de contrato, bonos colectivos (según establecimiento), años de experiencia, sueldo base, etc. Por otra parte, la ley establece que es responsabilidad del Estado complementar con fondos 
públicos la diferencia entre la remuneración anterior a la certificación por tramos y la nueva remuneración que algunos profesores recibirán, independiente del tipo de administración del establecimiento educativo (de administración municipal o de administración privada) esto evidencia -una vez más- el traspaso de fondos públicos para beneficios de privados.

Vemos también, que bajo este tipo de políticas subyace la concepción de docente visto como un técnico que ejecuta directrices orientadas por especialistas de la educación, que con cierta frecuencia lo evalúan y clasifican. Con respecto a esto, Cornejo (2006) postula que es más fácil para los docentes conseguir una mejora salarial que recuperar espacios de control sobre el proceso de trabajo educativo. Tanto así que en la educación pública chilena existen como mínimo cuatro niveles de decisión: el nivel central a cargo del Ministerio de Educación, las direcciones y supervisiones provinciales del Ministerio de Educación, las corporaciones y direcciones municipales de educación y, finalmente, la escuela. Por lo que los docentes están al final del proceso y su labor es simplemente la de ejecutar las políticas que emanan desde arriba.

De esta forma, la escuela es entendida desde los criterios de la productividad neoliberal en su relación eficacia-eficiencia para comprobar su rendimiento (MEJÍA, 2006), eso se traduce en presión constante para los docentes. Es decir, no existe democratización en la toma de decisiones educativas y para empeorar este escenario los profesores son sometidos a presiones en cuanto a rendimiento de sus estudiantes a través de pruebas estandarizadas que se utilizan como referentes mercantiles de promoción publicitaria para acreditar una mejor calidad educativa.

Este proceso que convierte el rol del docente en un ejecutor de las directrices que provienen de políticas ministeriales y municipales se denomina, según Mejía (2006) como despedagogización, centrando la profesión docente en la enseñabilidad, en donde la base de la acción educativa es la disciplina que se enseña, desde este enfoque la pedagogía sería solo las técnicas que hacen posible su instrumentalización. El profesorado por motivo de la despedagogización, dispone solo de los medios para la enseñanza y no de los fines que se persiguen con ésta (MORENO, 2013), por lo cual todo el trabajo intelectual lo realizan terceras personas. Junto al proceso de despedagogizacióno ocurre el de desprofesionalización en donde los docentes se convierten en operadores de la enseñanza a través de técnicas, los docentes dejan de tener un rol central en la educación pasando a ser un insumo más del proceso de enseñanza (MEJÍA, 2006). 
Vemos de esta forma que la Ley de CPD, al dejar fuera a los docentes de las decisiones con respecto a educación, evaluación docente y al desarrollo de la carrera profesional docente, consolida los procesos de despedagogización y desprofesionalización de la educación chilena obstaculizando una mejora en las condiciones laborales docentes.

Como vimos en el apartado anterior, la actual ley introdujo algunas reivindicaciones del movimiento docente, sin embargo, éstas fueron mínimas. Dentro de lo que no se consideró econtramos fuertes reivindicaciones que por años el gremio docente ha sostenido; nos referimos a bajar la cantidad de estudiantes por sala de clase, brindar una respuesta a la deuda histórica ${ }^{15}$, generar circunstancias que propicien un mayor cuidado de la salud docente y la creación de condiciones que disminuyan el agobio laboral. Como tampoco se tomó en cuenta a la crítica central del profesorado hacia el proyecto de 2015, la cual consistió es mostrar un fuerte rechazo a la lógica de evaluaciones y certificaciones periódicas. Por el contrario, como ya hemos mencionado, la ley de CDP genera un clima en que los profesores se sentirán aún más presionados al estar obligados a evaluárse continuamente, situación que genera más agobio laboral.

\section{Referencias}

\section{BIBLIOTECA NACIONAL DIGITAL. Conformación de la ideología neoliberal en Chile (1955-1978): Las siete modernizaciones. http://www.memoriachilena.cl/602/w3-article-31415.html\#links. Acceso en: 19 ago. 2015.}

CAMPAÑA POR UNA NUEVA EDUCACIÓN. Ideas para el Debate Colectivo. Por una Nueva Educación a Dignificar la Carrera Docente 2015. 2015. Disponible en: http://www.opech.cl/_doc/ideas_para_el_debate_colectivo.pdf Acceso en: 29 ago. 2015.

CHILE. Ley $\mathbf{N}^{\circ}$ 19.070, de 27 de junio de 1991. Estatuto de los Profesionales de la Educación.

\footnotetext{
${ }^{15}$ La Deuda Histórica tiene su origen en el incumplimiento de la Ley N ${ }^{\circ} 3.551$ de 1981 que estableció una asignación especial para los docentes dependientes del Ministerio de Educación. En el mismo año tras la municipalización la mayoría de los profesores no pudo recibir esta asignación en su integridad, ya que los nuevos empleadores no reconocieron tal derecho.
} 
. Ley $\mathrm{N}^{\circ} 19.410$, de 31 de agosto de 1995. Modifica la Ley $\mathrm{N}^{\circ} 19.070$, sobre Estatuto de Profesionales de la Educación, el decreto con fuerza de Ley $\mathrm{N}^{\circ} 5$, de 1993, del Ministerio de Educación, sobre subvenciones a establecimientos educacionales, y otorga beneficios que señala.

. Ministerio de educación. Revisión Nacional 2015 de la Educación para

Todos: Chile. 2014. Disponible en: http://www.acaoeducativa.org.br/desenvolvimento/wpcontent/uploads/2014/11/Informe Chile.pdf Acceso en: 20 jun. 2015.

Mensaje $N^{\circ}$ 165/363, de 20 de abril de 2015a. Mensaje de S.E. La Presidenta de la Republica con el que se inicia un Proyecto de Ley que crea el Sistema de Desarrollo Profesional Docente y Modifica otras Normas. Disponible en: $\quad$ http://www.bcn.cl/historiadelaley/nc/historia-de-la-ley/vistaexpandida/4890/\#h2 11 Acceso en: 10 ago. 2015.

. Ministerio de Educación. Estadísticas de la Educación 2014. Centro de Estudios MINEDUC. Santiago: 2015b. 157 p.

. Ministerio de Educación. Ley $\mathbf{N}^{\circ} \mathbf{2 0 . 9 0 3 , ~ d e ~} 4$ de marzo de 2016. Crea el Sistema de Desarrollo Profesional Docente y Modifica otras Normas. Santiago, 2016.

CORNEJO, Rodrigo. EI trabajo docente en la institución escolar. La apropiaciónenajenación del proceso de trabajo docente en el contexto de las reformas educativas neoliberales. Revista de Psicología, Santiago, Vol. XV, n. 2, p. 9-27, 2006.

ELACQUA, Gregory; SANTOS, Humberto. Preferencias reveladas de los proveedores de educación privada en Chile. El caso de la Ley de Subvención Escolar Preferencial. Revista Gestión y Política Pública, vol. XXII, núm. 1, 2013, pp. 85-129, primer semestre 2013. Disponible en: http://www.redalyc.org/articulo.oa?id=13325600003. Acceso en: 11 ago. 2015.

GONZALEZ, Luis. Arriba profes de Chile. De la precarización neoliberal a la reorganización docente. Santiago: Editorial América en Movimiento, 2015. 114 p.

. Los Desafíos del Profesorado ante el nuevo escenario. Revista Educación, Santiago, N¹1, p. 12- 14, may. 2016. 
LAMA, R. Carrera Profesional Docente del Gobierno: profundización del agobio y precariedad laboral. Revista Educación, Santiago, n9, p. 12- 14, jul. 2015.

MAYOL, Alberto; ARAYA, Javiera; AZÓCAR Carla; AZÓCAR Carlos. Fenómenos sobre educación y desigualdad., Santiago: Centro de Investigación en Estructura Socail - CIES [2011?]. Disponible en: http://hmart.cl/home/wpcontent/uploads/2011/03/DESIGUALDAD-Y-EDUCACION-INFORME-CIES-U-

DE-CHILE.pdf Acceso en: 27 ago, 2015.

MEJÍA, Marco Raúl. Cambio curricular y despedagogización en la globalización. Hacia una reconfiguración crítica de la pedagogía. Revista Docencia, Santiago, n. 28, p. 40- 53, may. 2006.

MINEDUC. Portal de Atención Ciudadana del Ministerio de Educación del Gobierno de Chile. Subvención escolar preferencial. Chile: [2017]. Disponível em: https://www.ayudamineduc.cl/ , acesso em 16/08/2017.

MÖNCKEBERG, María Olivia. El saqueo de los grupos económicos al Estado chileno. Chile: Ediciones B, 2001. 269 p.

MORENO, Karina, SOTO, Rodolfo. Políticas neoliberales en el contexto chileno: la profesión docente bajo la lógica mercantil. Revista Educere, Mérida, n. 58, p. 395401, sep. 2013.

MUD. Carrera Profesional Docente: Ley 20.903 «Crea el Sistema de Desarrollo Profesional Docente y Modifica otras normas». 2016. Disponible en: http://www.unidaddocente.cl/presentacion-sistema-desarrollo-profesionaldocente-cpd/Acceso en: 5 mar. 2017.

NÚÑEZ, Iván. Políticas hacia el Magisterio. In: GARCÍA-HUIDOBRO, Juan Eduardo. La Reforma Educacional Chilena. Madrid: Editorial Popular, 1999. Pp. 177-194. ; et alli. Las Transformaciones Educacionales bajo el Régimen Militar. Santiago, Chile: Programa Interdisciplinario de Investigaciones en Educación PIIE, Vol 1. 1984. Pp. 214.

OCDE. Revisión de Políticas Nacionales de Educación: Chile. París: OCDE, 2004. $304 \mathrm{p}$. 
OECD. Education at a Glande 2014: OECD Indicators. París: OCDE, 2014. Disponible en: http://www.oecd.org/education/Chile-EAG2014-CountryNote.pdf Acceso en: 1 jul. 2015.

. Education at a Glande 2015: OECD Indicators. París: OCDE, 2015. Disponible en: http://www.oecd.org/centrodemexico/medios/EAG2015_CN_CHL_ESP.pdf. Acceso en: 10 mar. 2017.

OPECH. Medir y castigar: la "no-pedagogía de la evaluación” en el proyecto de Carrera Profesional Docente. 2015. Disponible en: http://www.opech.cl/_doc/medir_y_castigar_evaluacion_cpd_documento.pdf Acceso en: 10 ago. 2015.

PARRA, Manuel. Estudio de Caso en Chile. In: KÖRNER, Anton, ROBALINO, Magaly. Condiciones laborales y salud docente: estudios de casos en Argentina, Chile, Ecuador, México, Perú y Uruguay. Santiago: UNESCO, 2005. Pp.75-102.

RUIZ, Carlos; BOCCARDO, Giorgio. Los chilenos bajo el neoliberalismo. Clases y conflicto social. Santiago de Chile: Editorial El buen aire, 2015. 186 p.

SENNETT, Richard. La Corrosión del Carácter. Las Consecuencias Personales del Trabajo en el nuevo Capitalismo. Traducción de Daniel Najmías. Barcelona: Editorial Anagrama, 2000.155 p.

SHIROMA, Eneida. Implicações da Política de Profissionalização Sobre a Gestão e o Trabalho Docente. Trabalho e Educação. Vol 13, №2. Ago -dez. 2004.

Enviado em 20/03/2017

Aprovado em 08/06/2017 
